\title{
Dopaminergic Suppression of Brain Deactivation Responses during Sequence Learning
}

\author{
Miklos Argyelan, ${ }^{1}$ Maren Carbon, ${ }^{1,2}$ Maria-Felice Ghilardi, ${ }^{3}$ Andrew Feigin, ${ }^{1,2}$ Paul Mattis, ${ }^{1,2}$ Chengke Tang, ${ }^{1,2}$ \\ Vijay Dhawan, ${ }^{1,2}$ and David Eidelberg ${ }^{1,2}$ \\ ${ }^{1}$ Center for Neurosciences, The Feinstein Institute for Medical Research and 2 Departments of Neurology and Medicine, North Shore University Hospital, \\ Manhasset, New York 11030, and ${ }^{3}$ Department of Pharmacology and Physiology, City University of New York Medical School, New York, New York 10031
}

Cognitive processing is associated with deactivation of the default mode network. The presence of dopaminoceptive neurons in proximity to the medial prefrontal node of this network suggests that this neurotransmitter may modulate deactivation in this region. We therefore used positron emission tomography to measure cerebral blood flow in 15 Parkinson's disease (PD) patients while they performed a motor sequence learning task and a simple movement task. Scanning was conducted before and during intravenous levodopa infusion; the pace and extent of movement was controlled across tasks and treatment conditions. In normal and unmedicated PD patients, learning-related deactivation was present in the ventromedial prefrontal cortex $(p<0.001)$. This response was absent in the treated condition. Treatment-mediated changes in deactivation correlated with baseline performance $(p<0.002)$ and with the $\mathrm{val}^{158} \mathrm{met}$ catechol-Omethyltransferase genotype. Our findings suggest that dopamine can influence prefrontal deactivation during learning, and that these changes are linked to baseline performance and genotype.

Key words: levodopa; Parkinson's disease; sequence learning; deactivation; default mode network; COMT genotype

\section{Introduction}

Motor sequence learning has been used increasingly to investigate the neural basis of skill acquisition in health and neurological disease. Functional imaging studies have revealed that this cognitive process is associated with a distinct activation pattern involving prefrontal, premotor, and posterior parietal cortical regions, as well as the basal ganglia (Nakamura et al., 2001; Carbon et al., 2003; Doyon, 2008). Like many goal-directed behaviors, motor sequence learning is also associated with deactivation in brain regions with pronounced metabolic activity in the rest state (Tamás Kincses et al., 2008). These areas, which include the medial prefrontal and parietal association regions, as well as the posterior cingulate cortex, comprise the default mode network (Raichle et al., 2001; Greicius et al., 2003). This spatially distributed neural system is thought to facilitate cognitive performance by reallocating neural resources to critical brain regions. Interestingly, the medial prefrontal node of this network receives input from the anterior cingulate area (Bates and Goldman-Rakic, 1993; Picard and Strick, 1996), a major locus of dopaminergic input to the cerebral cortex (Crino et al., 1993). L-Aromatic amino acid decarboxylase (AADC) is normally expressed in this brain area (Brown et al., 1999), and its expression is elevated in

Received June 24, 2008; revised Aug. 8, 2008; accepted Aug. 19, 2008.

This work was supported by National Institutes of Health-National Institute of Neurological Disorders and Stroke Grant R01 35069 (D.E.) and the General Clinical Research Center of The Feinstein Institute for Medical Research (Grant M01 RR018535). We thank Dr. Annette Lee for genotyping, Dr. Thomas Chaly for radiochemistry support, and Claude Margouleff for technical support. Special thanks to Toni Flanagan for valuable editorial assistance.

Correspondence should be addressed to Dr. David Eidelberg, Center for Neurosciences, The Feinstein Institute for Medical Research, 350 Community Drive, Manhasset, NY 11030. E-mail: david1@nshs.edu.

DOI:10.1523/JNEUROSCI.2933-08.2008

Copyright $\odot 2008$ Society for Neuroscience $\quad 0270-6474 / 08 / 2810687-09 \$ 15.00 / 0$ early stage Parkinson's disease (PD) patients (Rakshi et al., 1999; Kaasinen et al., 2000; Brück et al., 2005). Thus, levodopa can be decarboxylated and retained in dopaminergic terminals in this region.

We have found previously that levodopa can alter motor sequence learning performance and concurrent cortical activation responses in PD (Carbon et al., 2003; Feigin et al., 2003). We therefore investigated the possibility that this medication can influence task performance by dopaminergically modulating the medial prefrontal deactivation response. Positron emission tomography (PET) was used to measure cerebral blood flow (CBF) in $\mathrm{PD}$ patients while they performed a motor sequence learning task (SEQ) and a kinematically matched motor reference task. Scanning was conducted in an unmedicated baseline condition and repeated during intravenous levodopa infusion. In addition to assessing the effect of drug on learning-related deactivation, we determined how this response varied across subjects with differing baseline performance (Gibbs and D'Esposito, 2005; Cools et al., 2007). We also examined the relationship of intrinsic differences in prefrontal dopamine levels to local deactivation responses in both treatment conditions. This was achieved by correlating learning performance and associated neural responses with genotypic differences in the catechol-O-methyltransferase (COMT) val ${ }^{158}$ met polymorphism, which regulates dopamine metabolism in this brain region (Akil et al., 2003; MeyerLindenberg et al., 2005).

\section{Materials and Methods}

Subjects

We studied 15 right-handed PD patients [12 men and 3 women; age, $58.1 \pm 7.5$ years $($ mean $\pm S D)]$. The patients were chronically treated 
Table 1. Subject characteristics

\begin{tabular}{|c|c|c|c|c|c|c|c|c|c|c|}
\hline & \multirow[b]{2}{*}{ Age (years } & \multirow{2}{*}{$\begin{array}{l}\text { Hoehn- } \\
\text { Yahr stage }\end{array}$} & \multicolumn{2}{|c|}{ UPDRS (motor) ${ }^{a}$} & \multicolumn{2}{|l|}{ Onset time (ms) } & \multicolumn{2}{|c|}{ Movement time (ms) } & \multicolumn{2}{|c|}{ Retrieval index } \\
\hline & & & OFF & ON & OFF & ON & OFF & ON & OFF & ON \\
\hline PD & $58.1( \pm 7.5)$ & $2.3( \pm 0.7)$ & $25.1( \pm 7.5)$ & $16.3( \pm 5.2)$ & $-568( \pm 223)$ & $-595( \pm 220)$ & $592( \pm 132)$ & $516( \pm 109)$ & $11.9( \pm 7.3)$ & $11.5( \pm 9.4)$ \\
\hline \multicolumn{11}{|c|}{ Performance subgroups ${ }^{b}$} \\
\hline Good & $59.9( \pm 8.6)$ & $2.6( \pm 0.4)$ & $22.9( \pm 6.3)$ & $16.4( \pm 5.9)$ & $-554( \pm 154)$ & $-630( \pm 186)$ & $595( \pm 195)$ & $514( \pm 113)$ & $20.7( \pm 3.5)$ & $11.3( \pm 10.8)$ \\
\hline Bad & $55.2( \pm 4.1)$ & $2.0( \pm 0.6)$ & $25.0( \pm 5.7)$ & $16.2( \pm 3.4)$ & $-478( \pm 323)$ & $-583( \pm 265)$ & $536( \pm 87)$ & $517( \pm 113)$ & $6.3( \pm 2.3)$ & $14.1( \pm 9.7)$ \\
\hline \multicolumn{11}{|c|}{ Genotypic subgroups ${ }^{c}$} \\
\hline VAL & $54.1( \pm 7.9)$ & $2.1( \pm 0.8)$ & $20.9( \pm 3.9)$ & $12.3( \pm 2.5)$ & $-563( \pm 343)$ & $-663( \pm 218)$ & $593( \pm 87)$ & $548( \pm 77)$ & $10.9( \pm 6.5)$ & $15.4( \pm 12.2)$ \\
\hline MET & $53.6( \pm 2.6)$ & $1.9( \pm 0.6)$ & $23.6( \pm 6.1)$ & $15.0( \pm 3.1)$ & $-560( \pm 196)$ & $-663( \pm 286)$ & $534( \pm 84)$ & $505( \pm 109)$ & $6.9( \pm 3.1)$ & $11.7( \pm 11.0)$ \\
\hline
\end{tabular}

Data are shown as mean $( \pm S D)$.

a UPDRS items 19-31.

${ }^{b}$ Defined based on the distribution of baseline retrieval index (RET $\mathrm{OFF}_{\text {) }}$ ) values (see Materials and Methods).

'Defined by COMT val ${ }^{158}$ met haplotype (see Materials and Methods).

with oral levodopa/carbidopa, either alone or in combination with dopamine agonists. The demographic features of these patients are summarized in Table 1 . All subjects were scanned with $\mathrm{H}_{2}{ }^{15} \mathrm{O}$ PET, in an off state (OFF), defined as $12 \mathrm{~h}$ after the cessation of medication, and again in a stable on state $(\mathrm{ON})$ during an intravenous levodopa infusion. The details of the experimental design and the infusion protocol have been described previously (Feigin et al., 2003; Hirano et al., 2008). Antiparkinsonian medications were withheld for at least $12 \mathrm{~h}$ before each PET session. The patients were rated according to the motor portion of the Unified Parkinson's Disease Rating Scale (UPDRS; items 19-31) in the baseline OFF state and subsequently every $30 \mathrm{~min}$ during the levodopa infusion. Levodopa infusion rates were individually adjusted to achieve maximal improvement in motor ratings in a stable ON state without dyskinesia. The imaging experiments in the ON state were conducted in a steady state defined by $\leq 5 \%$ variation in motor ratings, and plasma levodopa levels recorded every $30 \mathrm{~min}$. In this cohort, levodopa infusion (mean rate, $0.83 \pm 0.76 \mathrm{mg} / \mathrm{kg} / \mathrm{hr}$; mean ON state plasma levodopa level, $13.94 \pm 8.1 \mu \mathrm{g} / \mathrm{ml})$ resulted in a stable improvement in UPDRS motor ratings (mean, $-34 \pm 12 \% ; p<0.001$ ). Nine right-handed healthy volunteer subjects (five men and four women; age, $55.9 \pm 10.8$ years) served as controls for the imaging studies.

The participants were nondemented and nondepressed with neuropsychological testing compatible with early/moderate PD (Asanuma et al., 2006). In the baseline OFF state, their scores were as follows: estimated intelligence quotient, $118 \pm 13$; Beck Depression Inventory, $5 \pm 3$; Brief Test of Attention, $7 \pm 2$; Hopkins Verbal Learning Test, $20 \pm 5$; delayed recall, $5 \pm 2$; symbol digit, $36 \pm 8$; word fluency, $44 \pm 14$. There was no visual evidence of atrophy or other structural brain abnormalities on magnetic resonance imaging (MRI). Ethical permission for all studies was obtained from the Institutional Review Board of North Shore University Hospital. Written consent was obtained from each subject after detailed explanation of the procedures.

\section{Behavioral testing}

Tasks. In each treatment condition, subjects performed two motor tasks during PET imaging: an SEQ and a kinematically matched motor execution control task (CCW) in which subjects moved to targets in a known counterclockwise order. The characteristics of these tasks have been described in detail previously (Nakamura et al., 2001; Ghilardi et al., 2003). Representative clips of the tasks can be viewed at http://feinsteinneuroscience.org/video/.

In performing both tasks, subjects moved a cursor on a digitizing tablet with the dominant right hand. Movements were out and back from a central starting position to one of eight radial targets displayed on the screen. In SEQ, the eight targets appeared in an unknown, pseudorandom order without repeating elements that was repeated over the $90 \mathrm{~s}$ trial block. Subjects were informed that a sequence was to be presented. They were instructed to learn the sequence order while reaching for the targets, to anticipate successive targets, and to reach each target in synchrony with a tone. Sequences were different in each trial run. For each SEQ run, a reaction time threshold for target anticipation was determined in a random reaction time task performed outside the scanner. In CCW, the eight targets appeared in a predictable counterclockwise order.
To reach the target in synchrony with the tone, subjects had to initiate movement before it appeared.

The pace of the movements in SEQ and CCW, represented by the intertone interval, was adapted to the subject's motor ability in the baseline OFF state, thereby experimentally fixing the rate of movement across tasks (SEQ, CCW) and treatment conditions (ON, OFF) (Feigin et al., 2003). The intertone interval was $1.5 \mathrm{~s}$ in 12 of the $15 \mathrm{PD}$ subjects, $2.0 \mathrm{~s}$ in two of the subjects and $1.83 \mathrm{~s}$ in one subject. Similarly, movement size was also held constant by adjusting the gain to a target extent of $1 \mathrm{~cm}$ for all subjects, tasks, and treatment conditions.

Performance measures. For SEQ, we computed the number of correct movements initiated below the reaction time threshold measured in the random reaction time task. These movements reflect anticipation and successful retrieval of previously acquired targets (Nakamura et al., 2001). The sum of all correctly anticipated targets in each trial block was defined as the retrieval index (RET) (Carbon et al., 2003; Ghilardi et al., 2003). This measure was used as the descriptor of the learning achieved in each SEQ trial. Levodopa-mediated changes in learning performance were assessed by comparing $\mathrm{RET}_{\mathrm{ON}}$ and $\mathrm{RET}_{\mathrm{OFF}}$ values using paired Student's $t$ tests. These changes were also correlated with baseline values $\left(\mathrm{RET}_{\mathrm{OFF}}\right)$ by calculating Pearson's product-moment correlation coefficients.

For CCW, we measured the mean onset time and movement time across cycles for each run (Carbon et al., 2007). The former measure can be considered the lead time before the movement start; the latter measurement is a reflection of movement speed. These values were correlated with the corresponding RET measures and were considered significant for $p<0.05$.

\section{Positron emission tomography}

All subjects fasted overnight before imaging. Regional CBF (rCBF) was estimated using $\mathrm{H}_{2}{ }^{15} \mathrm{O}$ PET in three-dimensional mode on the GE advance tomography at North Shore University Hospital (Nakamura et al., 2001). In the OFF and ON scan sessions, the SEQ and CCW tasks were performed in a randomized order with the dominant right arm. In 11 of the patients, the two tasks were performed twice. In the remaining four patients, the tasks were performed only once because of patient fatigue. In total, 24 complete scan sets (defined as paired SEQ and CCW scans acquired in both the ON and OFF states) were generated for additional analysis. The OFF state preceded the ON state in 16 sets; the ON state preceded the OFF state in the remainder.

Data processing was performed using SPM5 software (Wellcome Department of Cognitive Neurology, London, UK; http://www.fil.ion. ucl.ac.uk/spm/software/spm5/). Standard preprocessing (realignment, spatial normalizing, smoothing with $10 \times 10 \times 10 \mathrm{~mm}$ ) was applied followed by voxel-by-voxel comparisons using the flexible factorial model. SPM(t) maps were generated to assess the effects of task (SEQ, $\mathrm{CCW}$ ) and treatment condition (ON, OFF), and the interactions of these factors. In these analyses, RET was entered as a covariate of interest. To control for potential confounds resulting from variability in movement rate, the intertone interval was entered as a nuisance variable. 
Table 2. Regions with significant correlations between learning-related response and task performance

\begin{tabular}{|c|c|c|c|c|c|c|c|c|}
\hline \multirow[b]{2}{*}{ Brain region } & \multicolumn{3}{|c|}{ Coordinates $(\mathrm{mm})^{a}$} & \multirow[b]{2}{*}{$Z_{\max }$} & \multirow[b]{2}{*}{ Cluster size } & \multirow[b]{2}{*}{$p$ value $^{b}$} & \multicolumn{2}{|c|}{ Correlations $^{c}$} \\
\hline & $x$ & $y$ & $z$ & & & & $r$ & $p$ value \\
\hline \multicolumn{9}{|l|}{ Positive correlations } \\
\hline PARi, IPS, PARs (BA 40/7) & 36 & -74 & 28 & 3.73 & 375 & $0.031^{* *}$ & 0.51 & 0.000 \\
\hline Precuneus (BA 7) & -14 & -80 & 44 & 5.19 & 161 & $0.001^{* * *}$ & 0.48 & 0.000 \\
\hline preSMA/cingulate gyrus (BA 6/32) & 4 & 18 & 46 & 2.81 & 44 & $0.003^{*}$ & 0.39 & 0.005 \\
\hline DLPFC (BA 9) & 36 & 8 & 44 & 3.31 & 90 & $0.000^{*}$ & 0.36 & 0.011 \\
\hline \multicolumn{9}{|l|}{ Negative correlations } \\
\hline vmPFC (BA 10) & 0 & 50 & 6 & 3.39 & 56 & $0.000^{*}$ & -0.36 & 0.009 \\
\hline Inferior and middle temporal area (BA 20/21) & -58 & -10 & -20 & 2.88 & 89 & $0.002^{*}$ & -0.38 & 0.006 \\
\hline
\end{tabular}

PARi, Inferior parietal cortex; PARs, superior parietal cortex; IPS, intraparietal sulcus; DLPFC, dorsolateral prefrontal cortex.

${ }^{a}$ Coordinates are in Montreal Neurological Institute (MNI) space.

${ }^{b}$ Values at peak voxel within the hypothesis-testing mask (see Materials and Methods).

'Pearson product moment correlation coefficients ( $r$ ) and significance levels (p) for correlations between the RET and activation responses (SEQ - CCW) measured in VOls corresponding to each of the significant clusters.

*Values uncorrected for multiple comparisons; ${ }^{* *}$ false discovery rate corrected; ${ }^{* * *}$ family-wise error corrected.

Treatment-mediated changes in learning-related neural responses Regions with significant effects of levodopa on learning-related activation $($ SEQ $>$ CCW) or deactivation $($ SEQ $<$ CCW) were then classified based on whether treatment caused a loss, gain, or recovery of the data responses seen in healthy subjects. This was done by categorizing the CBF data measured in volumes of interest (VOIs) corresponding to the significant SPM clusters. For an individual VOI, an asterisk indicated a significant local learning-related neural response (i.e., SEQ $>\mathrm{CCW}$ or SEQ $<$ CCW; $p<0.05$, paired $t$ test); a dash indicated the absence of local response (i.e., no significant difference between the SEQ and CCW tasks). For each VOI, the learning-related response was signified by listing an asterisk or dash for the responses observed on each of the three conditions in order: the healthy controls first, followed respectively by the PD patients in the OFF and ON conditions. Thus, a "loss of response" was indicated by $\left({ }^{*},{ }^{*},-\right)$, reflecting a significant learning-related response in healthy controls and unmedicated PD patients, but not in levodopa-treated patients. A "gain of response" was indicated by $\left(-,-,{ }^{*}\right)$, reflecting a significant learning-related response in levodopa-treated PD patients. Last, a "recovery of response" was indicated by $\left(*,-,{ }^{*}\right)$, reflecting a significant learning-related response that was present in normal and treated PD patients, but not in unmedicated PD patients.

\section{Correlation between learning performance and associated neural responses}

Regions in which learning-related neural responses (activation, deactivation) correlated with task performance were identified in a voxelwise search. SPM correlational analysis was conducted within a predefined learning-specific brain volume (Feigin et al., 2003). In the present study, this mask (supplemental Fig. 1, available at www.jneurosci.org as supplemental material) was constructed from 104 SEQ and CCW scan pairs acquired in a separate population of $35 \mathrm{PD}$ patients scanned at baseline and 18 healthy volunteers (mean age, $54.1 \pm 15.6$ ). Discrete learningrelated spaces for activation (SEQ $>$ CCW) and deactivation $($ SEQ $<$ CCW) were specified at a voxel-level threshold of $p<0.001$, followed by correction for multiple comparisons at $p<0.05$. The individual regions comprising these spaces are presented in supplemental Table (available at www.jneurosci.org as supplemental material).

Voxel-wise correlations within the mask were considered significant at the hypothesis-testing threshold of $p<0.01$, uncorrected. An additional analysis used VOIs corresponding to the significant clusters in which local neural responses correlated with RET scores. Pearson product moment correlation coefficients were computed for each VOI, and were considered significant at $p<0.05$. In the significant regions, the VOI data were used post hoc to confirm that the correlations were not caused by interactions with the CCW motor task (Carbon et al., 2004). These VOIs were also used for hypothesis testing in the following subgroup analyses.

Predictors of levodopa-mediated alterations in learning responses Effect of baseline performance. The effect of baseline learning performance on levodopa-mediated changes in activation was further explored by contrasting good and bad learners. These patient subgroups were determined based on the distribution of the baseline learning scores $\left(\mathrm{RET}_{\mathrm{OFF}}\right)$, with good learners defined by performance in the upper tierce of values and bad learners by performance in the lower tierce (supplemental Fig. 2, available at www.jneurosci.org as supplemental material). Learningrelated neural responses in the two performance subgroups were first contrasted in the VOIs in which learning performance was found to correlate with local task-related activation (SEQ $>$ CCW) or deactivation $($ SEQ $<$ CCW) responses (Table 2).

In addition, we directly compared the SEQ and CCW scans obtained in the two performance subgroups by entering them into a flexible factorial model SPM to generate maps reflecting the effects of task (SEQ, CCW), treatment condition (OFF, ON), and performance subgroup (good, bad learners). In this exploratory analysis, the results were reported in the hypothesis-testing mask at $p<0.01$, uncorrected. This voxel-based method was used to compare the effects of levodopa on the activation responses across the two performance subgroups.

Regional interactions between subgroups and treatment condition were assessed in VOIs corresponding to the significant clusters detected in the SPM subgroup comparison, and in those identified in the correlation analysis described above. Learning-related neural responses (SEQ - CCW) were measured in each VOI and entered into a two-way repeated-measures ANOVA (RMANOVA) model with subgroup (good and bad learners) as the between-subject variable and condition (ON and OFF) as within-subject repeated measures. The results were considered significant at $p<0.05$.

Effect of the COMT val ${ }^{158}$ met genotype. Nine of the 15 subjects were retrospectively genotyped for the COMT val $^{158}$ met polymorphism to analyze the relationship of genotype to learning performance and levodopa-mediated changes in the related neural responses. The correlation between genotype (number of valine alleles) and mean baseline performance $\left(\mathrm{RET}_{\mathrm{OFF}}\right.$ ) across subjects was determined by computing the Spearman rank order correlation coefficient, which was considered significant for $p<0.05$. The effect of COMT haplotype on treatment-mediated changes in neural responses associated with learning was assessed using the same analytical strategy that was used to contrast the good and bad performance subgroups. The genotyped patients were divided into two subgroups: valine allele carriers (VAL) and methionine homozygotes (MET) (Meyer-Lindenberg et al., 2005). Interactions between subgroup (VAL, MET) and treatment condition (ON, OFF) were assessed as described above in the comparison of the good and bad learner performance subgroups. Data were analyzed in VOIs corresponding to the regions with significant activation/performance correlations as well as those with significant subgroup differences in treatment response. In these VOIs, the data were analyzed using two-way $2 \times 2$ RMANOVA and were considered significant at $p<0.05$. All statistical analyses were performed in SPSS. 


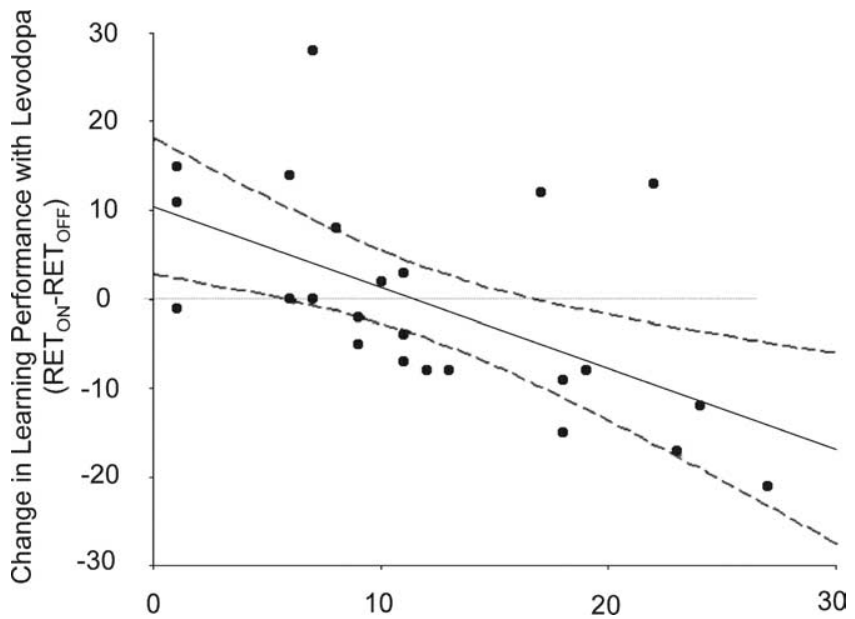

Figure 1. Sequence learning performance: relationship of levodopa-mediated changes to baseline values. Correlation between baseline performance ( $\mathrm{RET}_{\mathrm{OFF}}$ ) and the levodopamediated changes in learning ( $\left.\mathrm{RET}_{\mathrm{ON}}-\mathrm{RET}_{\mathrm{OFF}}\right)$. A significant negative correlation $(r=$ $-0.58, p=0.002$ ) was observed between these variables. The solid line is the best linear fit; dashed lines represent the $95 \%$ confidence interval.

Table 3. Regions with significant effects of levodopa on learning-related neural responses

\begin{tabular}{|c|c|c|c|c|c|c|}
\hline \multirow[b]{2}{*}{ Brain region } & \multicolumn{3}{|c|}{ Coordinates $(\mathrm{mm})^{a}$} & \multirow[b]{2}{*}{$Z_{\max }$} & \multirow[b]{2}{*}{ Cluster size } & \multirow[b]{2}{*}{$p$ value $^{b}$} \\
\hline & $x$ & $y$ & $z$ & & & \\
\hline \multirow{2}{*}{\multicolumn{7}{|c|}{ Levodopa-mediated changes in activation/deactivation response ${ }^{c}$}} \\
\hline Loss of response & & & & & & \\
\hline $\operatorname{vmPFC}(B A 10)$ & -4 & 52 & 0 & 4.14 & 259 & $<0.000$ \\
\hline Posterior insula & 48 & -14 & 4 & 4.21 & 839 & $<0.000$ \\
\hline Recovery of respons & & & & & & \\
\hline PMC (BA 6) & -22 & 0 & 60 & 5.08 & 516 & $<0.000$ \\
\hline $\begin{array}{l}\text { Gain of response } e^{d} \\
\text { preSMA (BA 6) }\end{array}$ & 14 & 6 & 72 & 3.57 & 47 & $<0.000$ \\
\hline
\end{tabular}

${ }^{a}$ Coordinates are in MNI space.

${ }^{b}$ Uncorrected values at peak voxel.

'See text for classification of the significant treatment effects.

${ }^{d}$ See supplementary Figure 3 (available at www.jneurosci.org as supplemental material) for illustration of the cerebral blood flow data in these regions.

\section{Results}

\section{Effects of levodopa on learning performance}

Group mean values $( \pm S D)$ for the learning and general movement performance measures in the two treatment conditions are presented in Table 1. There was no change in learning performance with treatment across the patient cohort $(p=0.85$, paired $t$ test $)$ and no effect of order with two treatment states $\left(F_{(1,23)}=\right.$ $0.46, p=0.50)$. Nonetheless, a significant inverse correlation $(r=$ $-0.58, p=0.002$ ) was evident between treatment-mediated changes in learning and baseline task performance (Fig. 1). Learning performance did not correlate with UPDRS motor ratings or with onset time and movement time indices measured during CCW in either treatment condition (OFF, $|r|<0.17, p>$ 0.42 ; ON, $|r|<0.15 ; p>0.46$ ).

\section{Effects of levodopa on learning-related neural responses} Treatment-mediated changes in regional activation/deactivation Regions with significant levodopa-mediated changes in learningrelated neural responses are presented in Table 3. Levodopa was associated with a loss of learning-related deactivation (SEQ $<$ $\mathrm{CCW}$ ) responses in the left ventromedial prefrontal cortex (vmPFC) (Fig. 2, top). A similar treatment-mediated loss of the deactivation response was detected in the right posterior insula, extending into the adjacent temporal cortex (Fig. 2, bottom). In both these areas, significant learning-related deactivation was present in healthy controls and unmedicated PD patients. This response was not present when the patients were scanned on levodopa.

Despite the loss of these deactivation responses with levodopa, no areas were identified in which learning-related activation $($ SEQ $>$ CCW) was reduced by treatment. Levodopa was, however, associated with increases in learning-related activation in the left dorsal premotor cortex [Brodmann's area (BA) 6] and in the right presupplemental motor area (pre-SMA) (Table 3, supplemental Fig. 3, available at www.jneurosci.org as supplemental material). In the former region, there was recovery of the normal activation response by levodopa. In the latter region, there was a treatment-mediated gain of response in that significant learningrelated activation was present only when the patients were scanned on levodopa therapy.

Correlation between learning performance and neural responses Regions in which learning-related (SEQ - CCW) activation responses correlated with task performance are presented in Table 2. Positive correlations between these measures were observed in the left precuneus (BA 7), and in the right dorsolateral prefrontal cortex (BA 46/9), inferior parietal lobule (BA 40), and the intraparietal sulcus (BA 40/7). Positive correlations were also present in the pre-SMA and anterior cingulate gyrus (BA 32). A significant negative correlation between learning and regional activation was observed in the vmPFC (BA 10), at coordinates similar to those associated with loss of deactivation during treatment (Table 3). A negative correlation was also identified in the inferior temporal gyrus (BA 20/21).

\section{Predictors of levodopa-mediated changes in learning responses}

Effect of baseline performance

We defined the top and bottom tierces of the distribution of RET scores at baseline as representing the good and bad performance subgroups (supplemental Fig. 2, shaded areas, available at www. jneurosci.org as supplemental material). The good learner subgroup $\left(\mathrm{RET}_{\mathrm{OFF}}, 17-27\right.$ ) was comprised of eight scan sets (SEQ CCW pairs in the OFF and ON conditions) from six patients. The bad learner subgroup ( RET $_{\text {OFF }}, 1-8$ ) was comprised of nine scan sets from seven patients. The clinical features of these subgroups are presented in Table 1 . The good and bad learners did not differ with respect to age, baseline disease severity, or change in motor UPDRS ratings with levodopa treatment $(p>0.19)$. The two performance subgroups also did not differ in the movement parameters (onset time and movement time) recorded during CCW performance $(p>0.22)$ or in neuropsychological performance at baseline $(p>0.17)$. The good and bad performers did however differ in the effect of levodopa on sequence learning $\left(F_{(1,15)}=7.92, p=0.01,2 \times 2\right.$ RMANOVA $)$. Post hoc testing revealed that levodopa improved performance in the bad learners ( $p=0.04$, paired $t$ test), and was associated with a trend toward worsening performance in the good learners $(p=0.09)$.

We next identified subgroup differences in the brain regions in which learning-related activity correlated with performance (see Materials and Methods). We found that the effect of levodopa on learning-related deactivation in the vmPFC differed for good and bad learners (Fig. $3 A$ ). For the good learners scanned in the unmedicated state, the deactivation response in this region resembled that of healthy subjects. Nonetheless, a significant reduction in this response occurred with levodopa treatment $(p=$ 

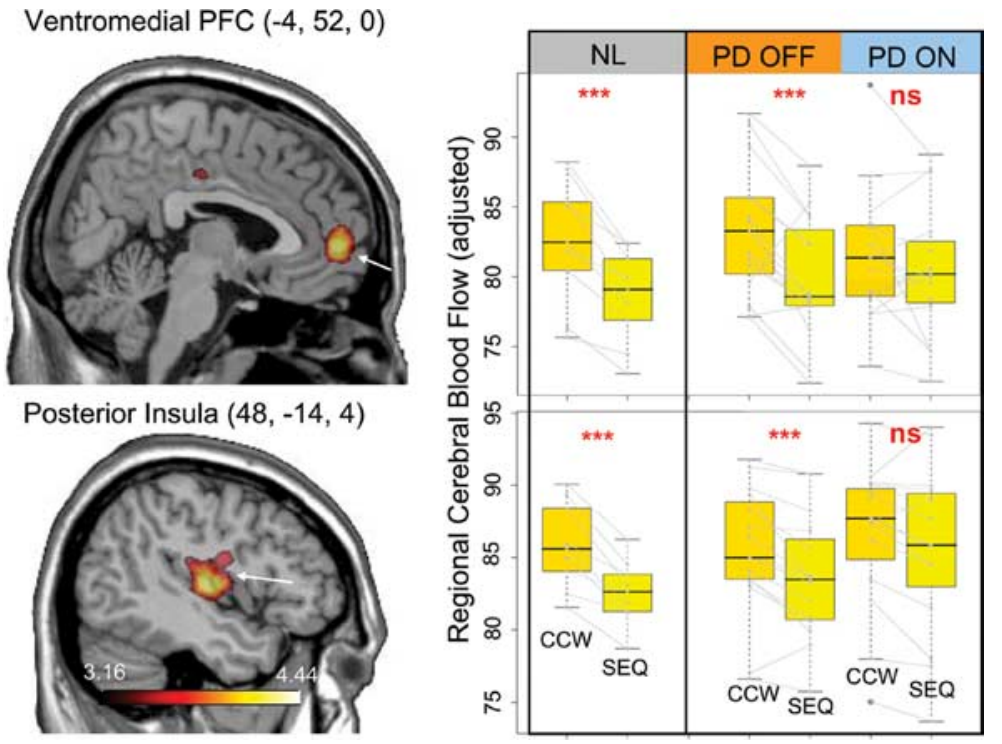

Figure 2. Changes in learning-related rCBF with levodopa infusion. Treatment was associated with loss of learning-related deactivation responses (SEQ $<C C W$ ) in the ventromedial prefrontal cortex (top) and the posterior insula (bottom). In both regions (arrows), significant deactivation during learning was present in healthy volunteers (NL) and unmedicated patients (PD OFF). These responses were not present in the same patients when they performed the sequence learning task during levodopa infusion (PD ON). Left, Voxel-based display of significant task (SEQ, CCW) by condition (OFF, ON) interaction effects on rCBF (SPM slices depict significant clusters at a threshold of $p<0.001$, uncorrected). Right, Post hoc analysis of rCBF data from V0ls corresponding to the clusters with significant interaction effects. Values for each task and condition are presented by box-and-whisker plots. ${ }^{* * *} p<0.001$, paired $t$ test. Paired $r C B F$ data across tasks for each subject are connected by lines.

0.04 , paired $t$ test). In contrast, baseline learning-related deactivation was minimal in the bad learners and was not altered by levodopa treatment $(p=0.97)$. Treatment-mediated changes in the other regions with significant correlations between performance and activation response did not differ across the two subgroups.

Direct voxel-based comparison of levodopa-mediated changes in activation across the performance subgroups disclosed a significant subgroup by treatment condition interaction effect in the precuneus $\left(8,-84,-26 ; Z_{\max }=4.22, p<0.001\right.$, uncorrected), which was confirmed on post hoc VOI analysis $\left(F_{(1,15)}=13.8, p=0.002 ; 2 \times 2\right.$ RMANOVA). In this region (Fig. $3 B)$, levodopa enhanced learning-related activation in bad learners $(p<0.05)$ while reducing this response $(p<0.01)$ in the good learners.

Effect of COMT val ${ }^{158}$ met genotype

Of the nine subjects who were genotyped for the COMT val ${ }^{158}$ met polymorphism (supplemental Fig. 2, color-coded data, available at www.jneurosci.org as supplemental material), four (seven scan sets) were met/met, four (seven scan sets) were $\mathrm{val} / \mathrm{met}$, and one (one scan set) was val/val. The clinical features of the val allele carriers (VAL) and the met homozygotes (MET) are presented in Table 1. These genotypic subgroups did not differ with respect to age, baseline disease severity, or the motor response to levodopa treatment $(p>0.27)$. They also did not differ in the CCW movement parameters $(p>0.21)$ or in baseline neuropsychological testing $(p>0.14)$. A trend toward positive correlation was present between baseline learning performance (RET ofF) and the number of valine alleles (Spearman's $r=0.475, p=0.09$ ), with relatively worse performance associated with met homozygosity.

Of the VOIs with significant correlations between learningrelated activation responses and performance, a significant inter- action between genotype and treatment condition was present only in the vmPFC (Fig. 4A). This effect was significant with or without including the val homozygote (all val allele carriers: $F_{(1,13)}=10.9, p<$ 0.01 ; val $/$ met only: $F_{(1,12)}=9.3, p<0.01$, $2 \times 2$ RMANOVA). In this region, levodopa reduced the magnitude of learningrelated deactivation in VAL $(p<0.05)$, although tended to enhance this response in $\operatorname{MET}(p=0.059)$.

Direct voxel-based comparison of scans from the VAL and MET subgroups revealed only one additional region with a significant genotype by treatment condition interaction effect. This cluster was localized to the cerebellar declive (lobule VI: $8,-84,-26 ; Z_{\max }=4.22, p<0.001$, uncorrected). This effect (Fig. $4 B$ ) was significant with or without including the $v a l$ homozygote (all val allele carriers: $F_{(1,13)}=$ 22.0, $p<0.0001$; val/met only: $F_{(1,12)}=$ $21.0, p<0.001,2 \times 2$ RMANOVA). In this region, levodopa reduced learning-related activation in VAL $(p<0.0001)$ while increasing this response in MET $(p<0.05)$. Post hoc testing also revealed that at baseline, learning-related activation in this area was significantly lower in the MET subgroup $(p<0.02)$.

\section{Discussion}

In this study, we show that levodopa can influence sequence learning performance through a loss of the cortical deactivation responses that are present in the unmedicated baseline state. The current findings revealed that levodopa specifically suppressed the normal deactivation response that occurs in the vmPFC during motor sequence learning (Tamás Kincses et al., 2008) (Fig. 2, top). The mechanism by which dopamine modulates the deactivation response is not known. We note that this region is closely associated with the ventral striatum (West and Grace, 2002; Grace et al., 2007). Dopaminergic input to this area is relatively preserved in $\mathrm{PD}$, perhaps rendering it more susceptible to local overdose effects (Cools, 2006). Alternatively, the effect of levodopa on learning-related vmPFC deactivation may be a consequence of increases in dopaminergic activity in the anterior cingulate cortex. Significant elevations in AADC activity have been noted in this region in PD patients undergoing ${ }^{18} \mathrm{~F}$-fluorodopa PET imaging (Rakshi et al., 1999; Kaasinen et al., 2001; Brück et al., 2005). Thus, systemically administered levodopa can be decarboxylated to dopamine in this region, resulting in potential overdose effects locally and in the adjacent medial prefrontal cortex.

These data suggest that learning-related deactivation in medial prefrontal cortex can be modulated by dopaminergic neurotransmission via the anterior cingulate cortex. Moreover, the observed treatment-mediated loss of deactivation responses in the insula, which is also closely connected with the anterior cingulate (Van Hoesen et al., 1993), may have occurred through a similar mechanism. These findings raise the possibility that, by analogy with the indirect striatopallidal pathway in the basal ganglia (Gerfen, 2000), $D_{2}$-like receptors on anterior cingulate neurons modulate output to projection targets in the neighboring medial pre- 


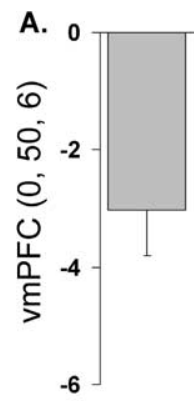

NL

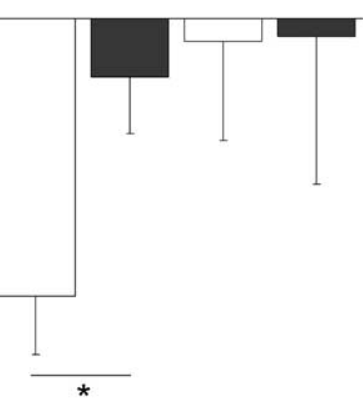

GOOD

BAD

Learning-related activation (SEQ-CCW)

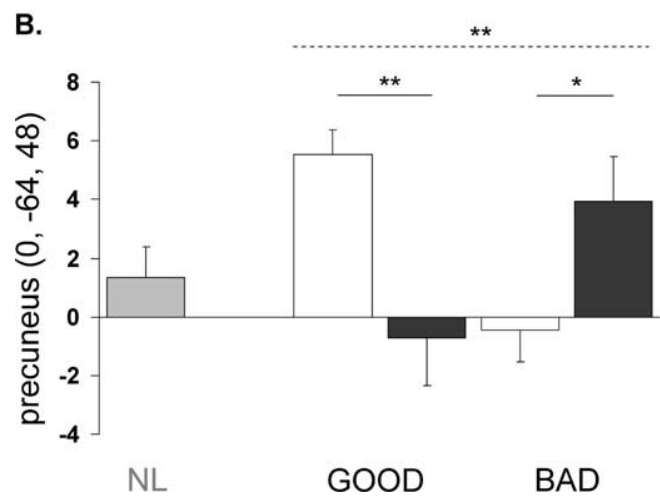

Figure 3. Effect of baseline performance on changes in learning-related neural responses with levodopa treatment. $\boldsymbol{A}$, Bar graph of learning-related deactivation (SEQ $<$ CCW) in the vmPFC for the patient subgroups with good and bad baseline performance (see Materials and Methods). In the unmedicated state (open bars), the good learners achieved a degree of deactivation that was comparable with healthy controls (shaded bar). In contrast, these responses were minimal in bad learners scanned at baseline. Neither of the two subgroups exhibited significant vmPFC deactivation in the treated state (filled bars). $\boldsymbol{B}$, Bar graph of learning-related activation (SEQ $>C C W$ ) in the precuneus for the good and bad performance subgroups. In this region, levodopa enhanced activation in bad learners, but attenuated this response in good learners. Parallel treatment-mediated behavioral effects were observed in the concurrently recorded learning performance data for the two subgroups (see Results). The dashed lines represent significant interaction effects $(2 \times 2$ RMANOVA). The solid lines represent significant pairwise differences (paired Student's t tests). ${ }^{*} p<0.05,{ }^{* *} p<0.01$. Error bars indicate SEM.

frontal cortex (Bates and Goldman-Rakic, 1993). $\mathrm{D}_{2}$ receptors are also present presynaptically on medial prefrontal cortex neurons that project to the ventral striatum (Okubo et al., 1999; Kaasinen et al., 2000; Grace et al., 2007; cf. Nikoshkov et al., 2008). Indeed, stimulation of these receptors has been found to reduce functional input to the latter region (West and Grace, 2002; Goto and Grace, 2005). Therefore, a predominantly $\mathrm{D}_{2}$ mediated pathway involving the anterior cingulate, medial prefrontal cortex, and ventral striatum potentially influence the default mode network by facilitating deactivation at its prefrontal node.

The relationship between levodopa-mediated changes in learning and baseline capability was further examined by comparing matched patient subgroups with good and bad performance levels in the untreated state. Using this approach, we found that in the good learners, vmPFC deactivation and concurrent task performance were normal at baseline, but declined with levodopa treatment. In contrast, the deactivation response was minimal in the bad learners, whether scanned at baseline or in the treated state. Dopaminergic therapy also affected activation responses in several parietal association regions. These areas corre-
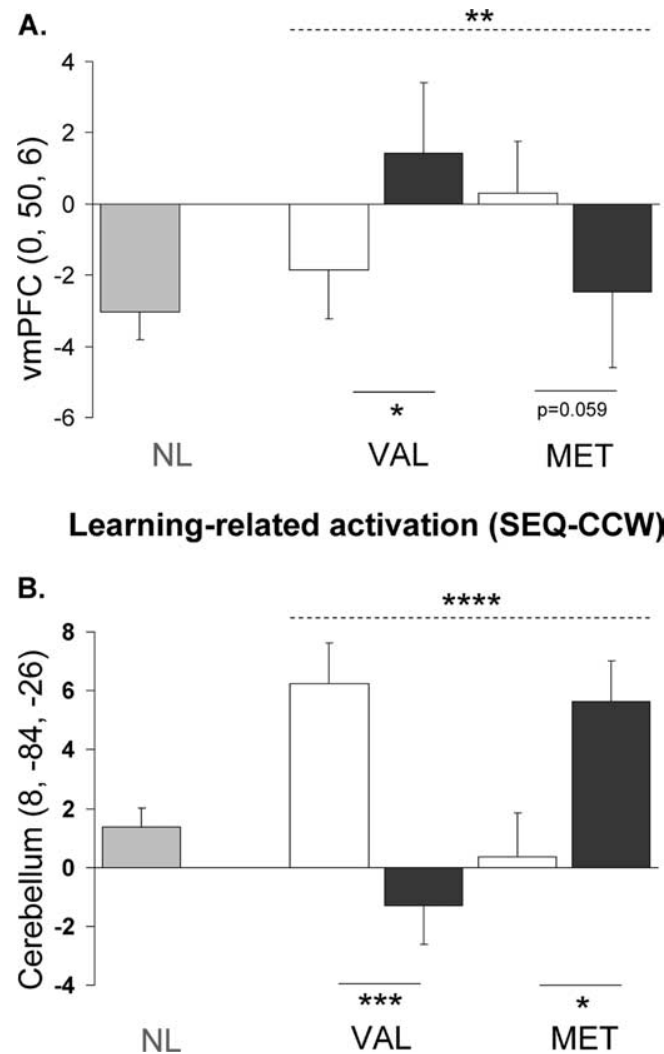

Figure 4. Effect of COMT genotype on changes in learning-related neural responses with levodopa treatment. $A$, Bar graph of learning-related deactivation (SEQ $<C C W$ ) in the vmPFC for patient subgroups with different COMT val ${ }^{158}$ met genotypes (see Materials and Methods). In this region, levodopa reduced the magnitude of deactivation in valine allele carriers, but enhanced this response in methionine homozygotes. $\boldsymbol{B}$, Bar graph of learning-related activation (SEQ $>$ CCW) in the cerebellum (lobule VI) for the two genotypic subgroups. In this region, levodopa reduced the magnitude of learning-related activation in valine allele carriers and enhanced in the methionine homozygotes. The baseline and treated conditions are represented by open and filled bars, respectively. The shaded bar represents reference values from healthy subjects. The dashed lines represent significant interaction effects $(2 \times 2$ RMANOVA). The solid lines represent significant pairwise differences (paired Student's $t$ tests). ${ }^{*} p<0.05$; ${ }^{* *} p<$ $0.01 ;{ }^{* * *} p<0.001 ;{ }^{* * * *} p<0.0001$. Error bars indicate SEM.

sponded topographically to the posterior cortical nodes of the normal learning-related activation network (Carbon et al., 2003). Indeed, in these regions, the activation responses paralleled the behavioral changes that were observed in the two performance subgroups. The findings suggest that the reduced levels of vmPFC deactivation seen in the bad learners can be compensated to some degree by the enhancement of learning-related activation responses in downstream network regions.

In addition to the influence of baseline phenotype on the cognitive response to dopaminergic therapy, we also found a significant influence of genotype. The observation that the effect of treatment on the vmPFC deactivation response varied according to the COMT val ${ }^{158}$ met genotype suggests that this physiologic effect is linked to intrinsic differences in prefrontal dopamine pools. Although only nine of the patients were available for DNA analysis, the results indicated a significant effect of genotype on the treatment-mediated changes in vmPFC deactivation. Specifically, in carriers of valine alleles, levodopa served to suppress learning-related deactivation in this region. In contrast, recovery of this response tended to occur in individuals without this allele (i.e., the methionine homozygotes). Interestingly, the individual data were found to conform to an inverted-U function (Fig. 5). This 


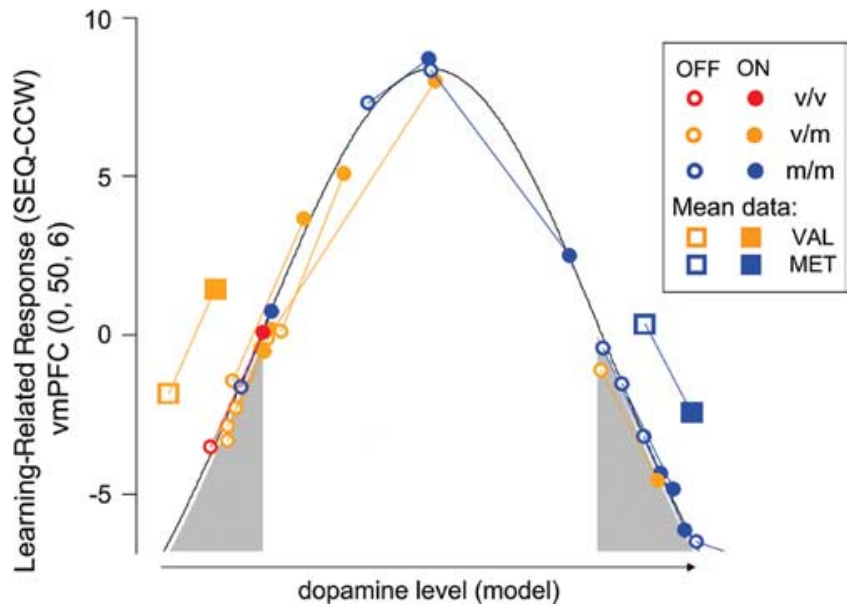

Figure 5. Learning-related deactivation responses in the vmPFC displayed as an inverted-U function. Bands of low and high dopamine at the edges of the curve (shaded areas) represent zones of optimal function in which local deactivation responses (SEQ - CCW $<0$ ) occur during task performance. The data were modeled using a simplex search method (Lagarias et al., 1998) to fit the measured learning-related responses to a Gaussian curve, $f(v)=p_{1} \times \exp \left(-(v)^{2} /\right.$ $\left.p_{2}\right)+p_{3}$, where $v$ is the hypothetical regional dopamine level and $f(v)$ is the corresponding activation response. The model was based on two major assumptions: (1) for all subjects, the local dopamine level was assumed to be higher in $0 \mathrm{~N}$ relative to $\mathrm{OFF}\left(v_{\mathrm{s}, \mathrm{ON}}>v_{\mathrm{s}, 0 \mathrm{FF}}\right.$, where $v_{i, \mathrm{j}}$ is the dopamine level for subject $i$ in condition $j$ ); and (2) because the valine allele is associated with greater activity of the enzyme in the brain (Chen et al., 2004), carriers of this allele were assumed to have relatively lower dopamine levels at baseline [mean $\left(v_{\text {val/val,off }}\right)<$ mean $\left(v_{\text {val/met, } 0 \mathrm{FF}}\right)<$ mean $\left.\left(v_{\text {met/met,ofF }}\right)\right]$. The model included two minor constraints: (1) the increment in dopamine level with treatment was of similar magnitude across genotypes $\left[\mathrm{SD}\left(v_{\mathrm{i}, 0 \mathrm{~N}}-\right.\right.$ $\left.v_{\mathrm{i}, 0 \mathrm{OFF}}\right)_{\mathrm{i} \in\{\text { all subjects }\}}$ is minimal]; and (2) the amplitude of $f(v)$ was determined by the range of the measured regional data. This fitting procedure was implemented in Matlab 6.5 (MathWorks).

type of nonlinear relationship between local neural activity and dopamine level has been documented consistently for task-related activation responses in dorsolateral PFC (Mattay et al., 2000; Goldberg and Weinberger, 2004). This, however, is the first demonstration of this inverted-U relationship involving a localized deactivation response. We note that, unlike the function observed with lateral PFC activation (Vijayraghavan et al., 2007), the peak of the current vmPFC curve corresponded to loss of the deactivation response (i.e., positive SEQ - CCW values). Indeed, a similar relationship has been recently reported in a functional MRI (fMRI) study of interactions between $\mathrm{D}_{2}$ receptor stimulation and genotype on activation responses during reversal learning (Cohen et al., 2007). This observation lends support to the hypothesis that vmPFC deactivation responses are modulated through $\mathrm{D}_{2}$ receptors in this cortical region, the ventral striatum, or both (Grace et al., 2007). Moreover, the difference between these inverted-U relationships, with enhancement or attenuation of neural activity at the peak, is consistent with opposing $\mathrm{D}_{1}$ - and $\mathrm{D}_{2}$-mediated effects on prefrontal functioning (Bilder et al., 2004; Seamans and Yang, 2004).

It is also of interest that, at baseline, better learning performance was associated with valine rather than methionine alleles. In healthy subjects, low-activity COMT genotypes like met/met involve increases in prefrontal dopamine levels and superior task performance (Malhotra et al., 2002). Nonetheless, this relationship may not apply to early stage PD patients in whom compensatory increases in dopaminergic input have been noted in lateral and medial PFC regions (Rakshi et al., 1999; Kaasinen et al., 2001; Brück et al., 2005). It has therefore been suggested that early stage PD patients are positioned on the descending limb of the classical inverted- $U$ function, with high activity COMT alleles (lower prefrontal dopamine) situated nearer to the peak (Foltynie et al., 2004). Indeed, this notion has been supported by recent fMRI studies in medicated patients in which valine homozygotes were found to have superior performance and prefrontoparietal activation than their methionine counterparts (Williams-Gray et al., 2007, 2008).

The current study revealed similarities and differences with these results. In agreement with the previous findings, methionine homozygotes were situated on the descending limb of the inverted-U curve. Likewise, the valine allele carriers fell to the left of the methionine homozygotes, although now they were on the ascending limb of the curve. As discussed above, individual measures of vmPFC deactivation conformed to a "nonclassical" inverted-U function, in which task-related neural responses were attenuated, rather than enhanced, in the neighborhood of the peak. Thus, optimal functioning was associated with discrete "bands" of dopamine activity (Fig. 5, shaded regions) situated on either side of the central region. This nonlinear relationship allowed for a deterioration of local deactivation responses in valine allele carriers during dopaminergic therapy, despite their positioning at baseline along the ascending portion of the curve.

We acknowledge that in valine allele carriers, the behavioral effects of treatment could also be explained by positioning along the descending limb of the classical inverted-U function, with a concomitant decline in learning-related activation responses (Williams-Gray et al., 2008). Nonetheless, this arrangement does not account for the treatment-mediated recovery of vmPFC deactivation seen in the methionine homozygotes, who presumably lie to the right of the valine allele carriers on this portion of the curve. This observation can, however, be explained by levodopa shifting these individuals further to the right into the band of optimal function on the extreme end of the curve.

We recognize that the behavioral data are not fully correlated with the neuroimaging findings in the vmPFC. Usually, more than one brain region accounts for the majority of individual differences in the performance of a complex task such as this (Nakamura et al., 2001; Carbon et al., 2003, 2004). As shown above (Fig. $3 B$ ), the parietal association cortex plays a major role in sequence learning performance. Thus, the vmPFC deactivations in themselves are unlikely to predict all or even most of the behavioral findings. That said, knowledge of the COMT genotype enhanced the understanding of the effects of levodopa on vmPFC function. Indeed, the data suggest that changes in deactivation in this region relate mainly to genotype, whereas parietal activation was determined mainly by baseline performance. Nonetheless, these measures are all interrelated to varying degrees.

We note that when present, vmPFC deactivation responses were associated with concurrent activation of the posteriormedial cerebellum. Thus, abnormally elevated cerebellar activation responses were evident in valine allele carriers scanned at baseline and in methionine homozygotes scanned in the treated condition. It is unlikely that these activations relate directly to levodopa treatment given the scarcity of AADCpositive terminals in this region. Moreover, enhanced cerebellar activation was also seen in valine allele carriers scanned in the unmedicated state. We speculate that the cerebellar response is associated with effective deactivation of the default mode network, as we suggest, occurs in the bands of optimal function situated at the edges of the inverted-U curve (see above). Suppression of activity within one or more nodes of this network would allow for a reallocation of neural resources to other learning-related regions, including the cerebellum.

The results of this study are limited by the relatively small 
sample size, especially with regard to inferences based on genotype. Nonetheless, we found that $\sim 30 \%$ of subject variation in the effect of levodopa on the vmPFC deactivation response could be predicted by combined knowledge of the COMT val ${ }^{158}$ met genotype and the baseline level of learning performance. It is additionally likely that predictive models of the cognitive levodopa response will become increasingly accurate as additional relevant polymorphisms are considered (Bertolino et al., 2006, 2008; Cohen et al., 2007). Likewise, it is possible that different dopamine genes selectively regulate the activity of the $D_{1}$ - and $\mathrm{D}_{2}$-mediated prefrontal-striatal pathways. Additional functional imaging studies using agonists for these receptors will help clarify the specific roles of these pathways in modulating cortical deactivation responses in health and disease.

\section{References}

Akil M, Kolachana BS, Rothmond DA, Hyde TM, Weinberger DR, Kleinman JE (2003) Catechol-O-methyltransferase genotype and dopamine regulation in the human brain. J Neurosci 23:2008-2013.

Asanuma K, Tang C, Ma Y, Dhawan V, Mattis P, Edwards C, Kaplitt MG, Feigin A, Eidelberg D (2006) Network modulation in the treatment of Parkinson's disease. Brain 129:2667-2678.

Bates JF, Goldman-Rakic PS (1993) Prefrontal connections of medial motor areas in the rhesus monkey. J Comp Neurol 336:211-228.

Bertolino A, Blasi G, Latorre V, Rubino V, Rampino A, Sinibaldi L, Caforio G, Petruzzella V, Pizzuti A, Scarabino T, Nardini M, Weinberger DR, Dallapiccola B (2006) Additive effects of genetic variation in dopamine regulating genes on working memory cortical activity in human brain. J Neurosci 26:3918-3922.

Bertolino A, Di Giorgio A, Blasi G, Sambataro F, Caforio G, Sinibaldi L, Latorre V, Rampino A, Taurisano P, Fazio L, Romano R, Douzgou S, Popolizio T, Kolachana B, Nardini M, Weinberger DR, Dallapiccola B (2008) Epistasis between dopamine regulating genes identifies a nonlinear response of the human hippocampus during memory tasks. Biol Psychiatry 64:226-234.

Bilder RM, Volavka J, Lachman HM, Grace AA (2004) The catechol-Omethyltransferase polymorphism: relations to the tonic-phasic dopamaine hypothesis and neuropsychiatric phenotypes. Neuropsychopharmacology 29:1943-1961.

Brown WD, Taylor MD, Roberts AD, Oakes TR, Schueller MJ, Holden JE, Malischke LM, DeJesus OT, Nickles RJ (1999) FluoroDOPA PET shows the nondopaminergic as well as dopaminergic destinations of levodopa. Neurology 53:1212-1218.

Brück A, Aalto S, Nurmi E, Bergman J, Rinne JO (2005) Cortical 6-[18F]fluoro-L-dopa uptake and frontal cognitive functions in early Parkinson's disease. Neurobiol Aging 26:891-898.

Carbon M, Ghilardi MF, Feigin A, Fukuda M, Silvestri G, Mentis MJ, Ghez C, Moeller JR, Eidelberg D (2003) Learning networks in health and Parkinson's disease: reproducibility and treatment effects. Hum Brain Mapp 19:197-211.

Carbon M, Ma Y, Barnes A, Dhawan V, Chaly T, Ghilardi MF, Eidelberg D (2004) Caudate nucleus: influence of dopaminergic input on sequence learning and brain activation in parkinsonism. Neuroimage 21:1497-1507.

Carbon M, Felice Ghilardi M, Dhawan V, Eidelberg D (2007) Correlates of movement initiation and velocity in Parkinson's disease: a longitudinal PET study. Neuroimage 34:361-370.

Chen J, Lipska BK, Halim N, Ma QD, Matsumoto M, Melhem S, Kolachana BS, Hyde TM, Herman MM, Apud J, Egan MF, Kleinman JE, Weinberger DR (2004) Functional analysis of genetic variation in catechol-Omethyltransferase (COMT): effects on mRNA, protein, and enzyme activity in postmortem human brain. Am J Hum Genet 75:807-821.

Cohen MX, Krohn-Grimberghe A, Elger CE, Weber B (2007) Dopamine gene predicts the brain's response to dopaminergic drug. Eur J Neurosci 26:3652-3660.

Cools R (2006) Dopaminergic modulation of cognitive functionimplications for L-DOPA treatment in Parkinson's disease. Neurosci Biobehav Rev 30:1-23.

Cools R, Sheridan M, Jacobs E, D’Esposito M (2007) Impulsive personality predicts dopamine-dependent changes in frontostriatal activity during component processes of working memory. J Neurosci 27:5506-5514.

Crino PB, Morrison JH, Hof PR (1993) Monoaminergic innervation of cingulate cortex. In: Neurobiology of cingulate cortex and limbic thalamus: a comprehensive handbook (Vogt BA, Gabriel M, eds), pp 285-310. Boston: Birkhauser.

Doyon J (2008) Motor sequence learning and movement disorders. Curr Opin Neurol 24:478-483.

Feigin A, Ghilardi MF, Carbon M, Edwards C, Fukuda M, Dhawan V, Margouleff C, Ghez C, Eidelberg D (2003) Effects of levodopa on motor sequence learning in Parkinson's disease. Neurology 60:1744-1749.

Foltynie T, Goldberg TE, Lewis SG, Blackwell AD, Kolachana BS, Weinberger DR, Robbins TW, Barker RA (2004) Planning ability in Parkinson's disease is influenced by the COMT val158met polymorphism. Mov Disord 19:885-891.

Gerfen CR (2000) Molecular effects of dopamine on striatal-projection pathways. Trends Neurosci 23:S64-70.

Ghilardi MF, Eidelberg D, Silvestri G, Ghez C (2003) The differential effect of PD and normal aging on early explicit sequence learning. Neurology 60:1313-1319.

Gibbs SE, D'Esposito M (2005) Individual capacity differences predict working memory performance and prefrontal activity following dopamine receptor stimulation. Cogn Affect Behav Neurosci 5:212-221.

Goldberg TE, Weinberger DR (2004) Genes and the parsing of cognitive processes. Trends Cogn Sci 8:325-335.

Goto Y, Grace AA (2005) Dopaminergic modulation of limbic and cortical drive of nucleus accumbens in goal-directed behavior. Nat Neurosci 8:805-812.

Grace AA, Floresco SB, Goto Y, Lodge DJ (2007) Regulation of firing of dopaminergic neurons and control of goal-directed behaviors. Trends Neurosci 30:220-227.

Greicius MD, Krasnow B, Reiss AL, Menon V (2003) Functional connectivity in the resting brain: a network analysis of the default mode hypothesis. Proc Natl Acad Sci U S A 100:253-258.

Hirano S, Asanuma K, Ma Y, Tang C, Feigin A, Dhawan V, Carbon M, Eidelberg D (2008) Dissociation of metabolic and neurovascular responses to levodopa in the treatment of Parkinson's disease. J Neurosci 28:4201-4209.

Kaasinen V, Nagren K, Hietala J, Oikonen V, Vilkman H, Farde L, Halldin C, Rinne JO (2000) Extrastriatal dopamine D2 and D3 receptors in early and advanced Parkinson's disease. Neurology 54:1482-1487.

Kaasinen V, Nurmi E, Brück A, Eskola O, Bergman J, Solin O, Rinne JO (2001) Increased frontal [18F]fluorodopa uptake in early Parkinson's disease: sex differences in the prefrontal cortex. Brain 124:1125-1130.

Lagarias JC, Reeds JA, Wright MH, Wright PE (1998) Convergence properties of the Nelder-Mead simplex method in low dimensions. SIAM J Optimization 9:112-147.

Malhotra AK, Kestler LJ, Mazzanti C, Bates JA, Goldberg T, Goldman D (2002) A functional polymorphism in the COMT gene and performance on a test of prefrontal cognition. Am J Psychiatry 159:652-654.

Mattay VS, Callicott JH, Bertolino A, Heaton I, Frank JA, Coppola R, Berman KF, Goldberg TE, Weinberger DR (2000) Effects of dextroamphetamine on cognitive performance and cortical activation. Neuroimage 12:268-275.

Meyer-Lindenberg A, Kohn PD, Kolachana B, Kippenhan S, McInerney-Leo A, Nussbaum R, Weinberger DR, Berman KF (2005) Midbrain dopamine and prefrontal function in humans: interaction and modulation by COMT genotype. Nat Neurosci 8:594-596.

Nakamura T, Ghilardi MF, Mentis M, Dhawan V, Fukuda M, Hacking A, Moeller JR, Ghez C, Eidelberg D (2001) Functional networks in motor sequence learning: abnormal topographies in Parkinson's disease. Hum Brain Mapp 12:42-60.

Nikoshkov A, Drakenberg K, Wang X, Horvath MC, Keller E, Hurd YL (2008) Opioid neuropeptide genotypes in relation to heroin abuse: dopamine tone contributes to reversed mesolimbic proenkephalin expression. Proc Natl Acad Sci U S A 105:786-791.

Okubo Y, Olsson H, Ito H, Lofti M, Suhara T, Halldin C, Farde L (1999) PET mapping of extrastriatal D2-like dopamine receptors in the human brain using an anatomic standardization technique and [11C]FLB 457. Neuroimage 10:666-674.

Picard N, Strick PL (1996) Motor areas of the medial wall: a review of their location and functional activation. Cereb Cortex 6:342-353. 
Raichle ME, MacLeod AM, Snyder AZ, Powers WJ, Gusnard DA, Shulman GL (2001) A default mode of brain function. Proc Natl Acad Sci U S A 98:676-682.

Rakshi JS, Uema T, Ito K, Bailey DL, Morrish PK, Ashburner J, Dagher A, Jenkins IH, Friston KJ, Brooks DJ (1999) Frontal, midbrain and striatal dopaminergic function in early and advanced Parkinson's disease a 3D [18F]dopa-PET study. Brain 122:1637-1650.

Seamans JK, Yang CR (2004) The principal features and mechanisms of dopamine modulation in the prefrontal cortex. Prog Neurobiol 74:1-58.

Tamás Kincses Z, Johansen-Berg H, Tomassini V, Bosnell R, Matthews PM, Beckmann CF (2008) Model-free characterization of brain functional networks for motor sequence learning using fMRI. Neuroimage 39:1950-1958.

Van Hoesen GW, Morecraft RJ, Vogt BA (1993) Connections of the monkey cingulate cortex. In: Neurobiology of cingulate cortex and limbic thalamus: a comprehensive handbook (Vogt BA, Gabriel M, eds), pp 249-284. Boston: Birkhauser.

Vijayraghavan S, Wang M, Birnbaum SG, Williams GV, Arnsten AF (2007) Inverted- $\mathrm{U}$ dopamine $\mathrm{D} 1$ receptor actions on prefrontal neurons engaged in working memory. Nat Neurosci 10:376-384.

West AR, Grace AA (2002) Opposite influences of endogenous dopamine D1 and D2 receptor activation on activity states and electrophysiological properties of striatal neurons: studies combining in vivo intracellular recordings and reverse microdialysis. J Neurosci 22:294-304.

Williams-Gray CH, Hampshire A, Robbins TW, Owen AM, Barker RA (2007) Catechol O-methyltransferase Val158Met genotype influences frontoparietal activity during planning in patients with Parkinson's disease. J Neurosci 27:4832-4838.

Williams-Gray CH, Hampshire A, Barker RA, Owen AM (2008) Attentional control in Parkinson's disease is dependent on COMT val 158 met genotype. Brain 131:397-408. 